\title{
Neues Medizinalberufegesetz: schlanke Regelung der ärztlichen Berufsbildung
}

Am 1. September 2007 wird das Freizügigkeitsgesetz für Medizinalberufe, das zum Schutz der Bevölkerung vor Scharlatanerie 1877 in Kraft gesetzt wurde, durch das Medizinalberufegesetz (MedBG) abgelöst. Dieses regelt die bisherigen vier universitären Medizinalberufe in den Bereichen Medizin, Zahnmedizin, Tiermedizin, Pharmazie sowie neu und in Europa einmalig als fünften universitären Medizinalberuf den Bereich Chiropraktik.

Das MedBG regelt im Interesse der öffentlichen Gesundheit die Qualität der Aus-, Weiter- und Fortbildung. Es definiert im Bereich des Arztberufes allgemeine Grundsätze sowie Verfahrensfragen und legt die Ziele für eine eigenverantwortliche ärztliche Berufsausübung fest. Diese Ziele werden durch einen kontinuierlichen, inhaltlich abgestimmten Lernprozess, beginnend mit der universitären Ausbildung, gefolgt von der berufsbegleitenden Weiterbildung bis zur lebenslangen Fortbildung, erreicht. Der Bund überwacht das Erreichen der Ziele von Ausbildung und Weiterbildung mittels Akkreditierung der universitären Ausbildungsgänge und der Weiterbildungsprogramme. Deren detaillierte Ausgestaltung und Umsetzung wird der Autonomie der medizinischen Fakultäten beziehungsweise der FMH überlassen.
Als Novum regelt das MedBG die Berufspflichten für alle selbständigtätigen Ärztinnen und Ärzte in der Schweiz. Dazu gehört die Pflicht zur Fortbildung während der aktiven Berufsausübung.

Das MedBG ermöglicht eine schlanke und effiziente staatliche Aufsicht über die von den Berufsorganisationen geregelte Berufsbildung. Dieses System bewährt sich bereits seit dem 1. Juni 2002, Zeitpunkt des Inkrafttretens der bilateralen Verträge mit der EU, auch wenn sich der Bund zunehmend in ärztliche Bildungsbelange einmischt.

Die Planung und der Ablauf der nächsten Akkreditierung der 44 ärztlichen Weiterbildungsprogramme, die gemäss MedBG im Herbst 2011 stattfinden soll, wird zum Prüfstein für das Bundesamt für Gesundheit (BAG) bezüglich der Effizienz und des Willens zur partnerschaftlichen Zusammenarbeit mit der FMH.

Dr. med. Max Giger, Mitglied des Zentralvorstandes der FMH, Präsident Kommission für Weiter- und Fortbildung der FMH 